\title{
The Effects of Burst Activity on Soft Gamma Repeater Pulse Properties and Persistent Emission
}

\author{
Peter M. Woods \\ Universities Space Research Association \\ National Space Science and Technology Center \\ 320 Sparkman Dr., Huntsville, AL 35805
}

\begin{abstract}
.
Soft Gamma Repeaters (SGRs) undergo changes in their pulse properties and persistent emission during episodes of intense burst activity. SGR $1900+14$ has undergone large flux increases following recent burst activity. Both SGR 1900+14 and SGR 1806-20 have shown significant changes in their pulse profile and spindown rates during the last several years. The pulse profile changes are linked with the burst activity whereas the torque variations are not directly correlated with the bursts. Here, we review the observed dynamics of the pulsed and persistent emission of SGR 1900+14 and SGR 1806-20 during burst active episodes and discuss what implications these results have for the burst emission mechanism, the magnetic field dynamics of magnetars, the nature of the torque variability, and SGRs in general.
\end{abstract}

\section{INTRODUCTION}

Soft Gamma Repeaters (SGRs) are an exotic class of high energy transient, very likely isolated, strongly magnetized neutron stars or "magnetars." For periods of days to months, SGRs can be found in burst active states where they emit anywhere from a handful to several hundred bursts. Typically, the bursts last $\sim 0.1 \mathrm{sec}$ and have energy spectra $(E>25 \mathrm{keV})$ that can be modeled as a power-law convolved with an exponential. At lower energies, however, this empirical model fails to fit the spectrum [1]. The burst energies follow a power-law number distribution up to $\sim 10^{42} \operatorname{ergs}\left(\mathrm{dN} / \mathrm{dE} \propto \mathrm{E}^{-5 / 3}[2,3]\right)$, consistent with a so-called self-organized critical system (e.g. earthquakes, Solar flares, etc. [4]) where the burst energy resevoir greatly exceeds the energy output within any given burst. On two occasions, more energetic bursts or giant flares were recorded from SGR 0526-66 on 1979 March 5 [5] and SGR 1900+14 on 1998 August $27[6,7,8,9]$. Each of these extraordinary events had a bright $\left(\sim 10^{44} \mathrm{ergs} \mathrm{s}^{-1}\right)$, spectrally hard initial spike followed by a softer, several minute long tail showing coherent pulsations at 8 and $5 \mathrm{~s}$, respectively. More recently, an intermediate flare $\left(\sim 10^{43} \mathrm{ergs}\right)$ lasting $40 \mathrm{~s}$ was recorded from SGR 1900+14 on 2001 April 18 [10].

All SGRs are associated with persistent X-ray counterparts; three of them have quiescent luminosities $\sim 10^{34}$ $\operatorname{ergs~s}^{-1}$, while the quiescent flux level of SGR 1627-41 has not yet been determined [11]. The spectra of three
SGRs can be modeled with a power-law (photon indices $\sim 2-3.5$ ); SGR $1900+14$ requires an additional blackbody component $(k T \sim 0.5 \mathrm{keV}[12,13,14])$. Two SGRs show low-amplitude pulsations in their persistent emission. The frequency of these pulsations is increasing rapidly, consistent with the interpretation of an underlying strongly magnetized neutron star [15]. For a more comprehensive review of the properties of SGRs, see [16].

During the last few years, changes in the X-ray emission properties of SGRs have been noted during episodes of burst activity $[13,17]$. Through studying the transient effects imparted upon SGRs (or the lack thereof) during times of burst activity, we have gained deeper insight into the nature of the burst mechanism and the SGR systems in general. Here, we review the observed influence of burst activity on SGR pulse properties and persistent X-ray emission, limiting ourselves to the two SGRs that show pulsations in their X-ray emission, namely SGR 1900+14 and SGR 1806-20.

\section{PERSISTENT AND PULSED FLUX}

Changes in the flux of SGRs was first noted in SGR $1900+14$ following the giant flare of August $27[18,19,20,12]$. Following this discovery, a compilation of persistent and pulsed flux measurements over several years (Figure 1 [13]) revealed that, in general, 

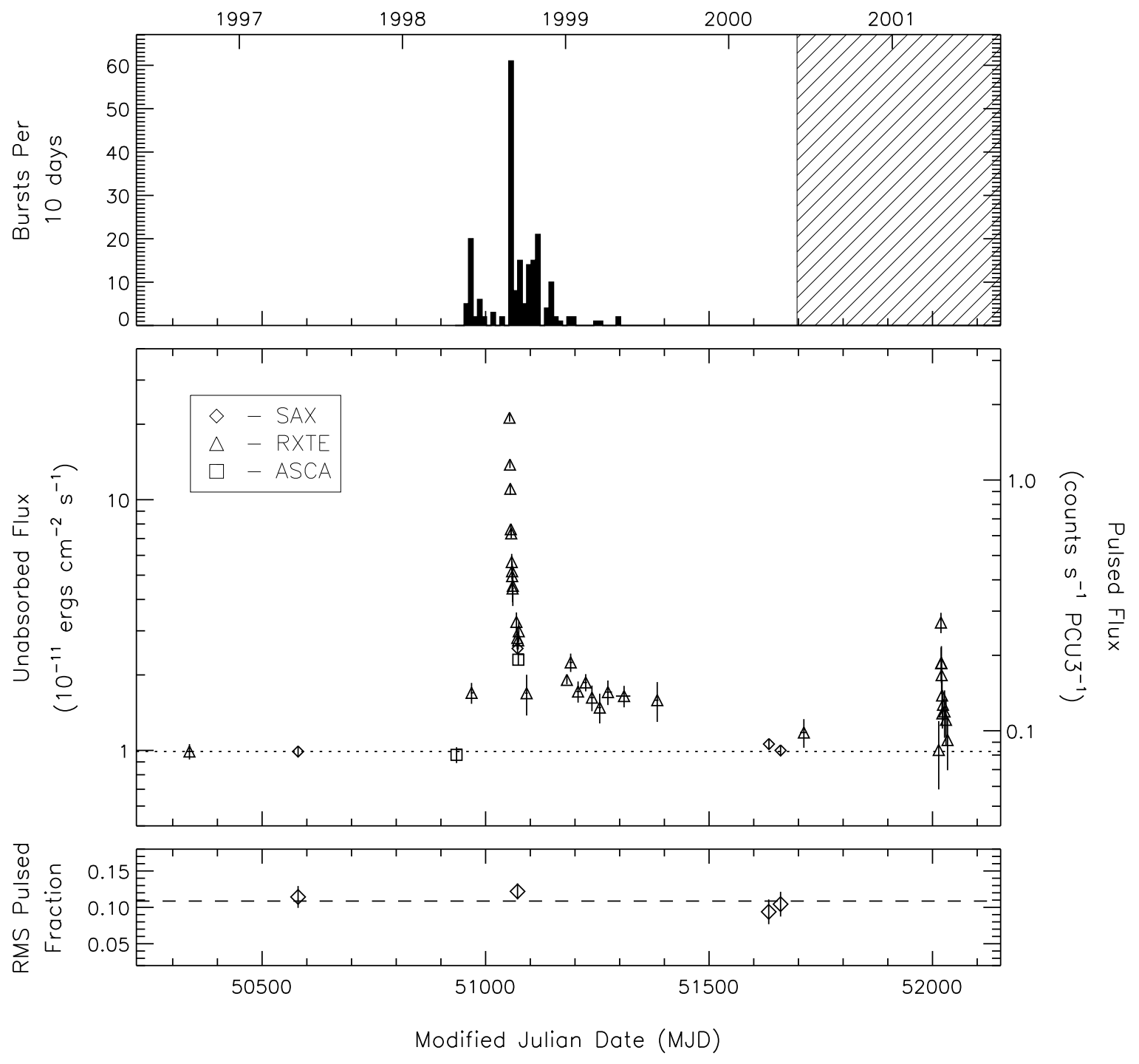

FIGURE 1. Top panel - Burst rate history of SGR 1900+14 as observed with BATSE. Middle panel - Persistent/Pulsed flux history of SGR $1900+14$ covering 4.5 years. The left vertical scale is unabsorbed $2-10 \mathrm{keV}$ flux and the right is pulsed flux in units of counts $\mathrm{s}^{-1} \mathrm{PCU}^{-1}$. The dotted line marks the nominal quiescent flux level of this SGR. Note that the spike in the pulsed flux shortly after MJD 52000 coincides with a burst active episode not covered by the BATSE monitoring (top). See text for further details. Bottom panel - Pulse fraction of SGR 1900+14 $(2-10 \mathrm{keV})$ as measured within the four BeppoSAX observations using the MECS instruments. The dashed line marks the mean RMS pulsed fraction $\left(f_{\text {RMS }} \sim 0.11\right)$. 
there is an excellent correlation between burst activity (top) and enhancements in the persistent/pulsed flux from this SGR (middle). We have found that the pulse fraction (bottom) is consistent with remaining constant at most epochs despite changes in the persistent flux. It is by assuming that this fraction remains constant at all times that we can plot both the pulsed flux (RXTE PCA) and the persistent flux (BeppoSAX and ASCA) on the same scale. We note, however, that there are exceptions to this rule when the pulse fraction has increased for short periods of time (see below).

We have found that the brightest pulsed/persistent flux excess seen in Figure 1 is directly linked with the August 27 flare. The excess decays approximately as a powerlaw in time $\left(F \propto t^{-0.7}\right)$ following the giant flare (Figure 2 [13]), qualitatively similar to GRB afterglows. In order to avoid confusion between the two phenomena, we will refer to the excesses in SGRs as X-ray tails rather than afterglows hereafter. The spectrum $(0.1-10 \mathrm{keV})$ of the $\mathrm{X}$-ray tail at $\sim 19$ days after the flare was found to be exclusively non-thermal [12].

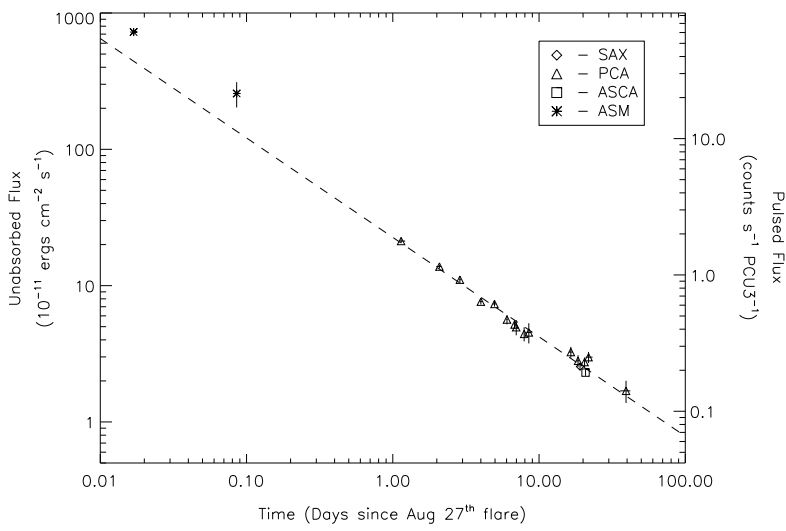

FIGURE 2. The flux decay following the 1998 August 27 flare from SGR $1900+14$. The reference time is the beginning of the flare as observed in soft $\gamma$-rays. The dotted line is a fit to the RXTE/PCA, BeppoSAX, and ASCA data only (i.e. the ASM data are not included in the fit). The slope of this line is $-0.713 \pm 0.025$.

For SGR $1900+14$, there are now four X-ray tails that can be linked with specific bursts or flares. The second of these events was recorded on 1998 August 29 (Figure 3 [17]). This burst had a high gamma-ray fluence and an X-ray tail whose bolometric flux decayed approximately as a power-law in time. The spectrum of this tail softens with time. Formally, the spectrum is equally well fit by a power-law plus a blackbody or a thermal bremsstrahlung, each with interstellar attenuation [17]. However, the bremsstralung model yields a column density $\sim 5$ times larger than the measured column from the persistent emission whereas the two component model fit yields a column consistent with the persistent emission value. The pulsed fraction increases above the quiescent level (11\% RMS) up to $\sim 20 \%$ during this tail (Figure 4 [21]), and the phase of the pulsations do not shift during the tail relative to the pre-burst pulse phase.

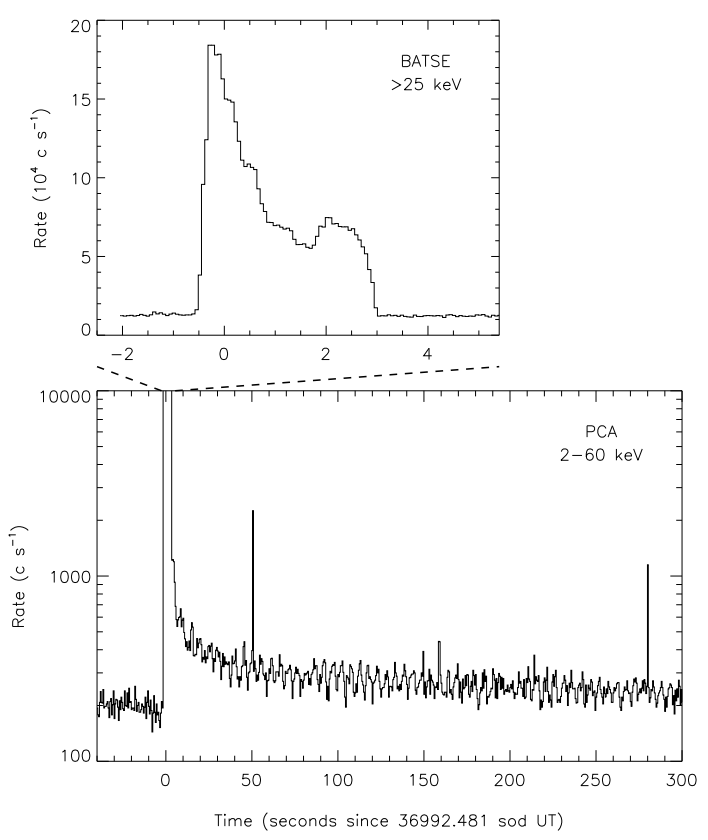

FIGURE 3. The energetic burst of 1998 August 29 from SGR $1900+14$ as seen with BATSE (top panel) and the RXTE PCA (bottom panel).

The last two bursts with X-ray tails were detected on 2001 April 18 and April 28. Spectral analysis of the April 18 burst tail is presented in [14, 22, 23]. The April 28 event is discussed in greater detail elsewhere in this volume [21]. During each of these events, the pulse fraction was found to increase during the tail [24, 21]. The spectrum of the April 28 tail is a cooling blackbody [21], dissimilar to the 1998 August tails which each required a power-law component.

Even though we have detected just four X-ray tails following energetic bursts from SGR 1900+14, we find significant differences between them. First, there are varying levels of thermal and non-thermal emission within the tails. Also, the pulse fraction increases by up to a factor $\sim 3$ in one case (April 28) and not at all in another (August 27). An interesting trend which arises from this small set of X-ray tails is that the pulse fraction enhancement in the separate X-ray tails appears to correlate with the magnitude of the thermal contribution to the X-ray flux. That is, tails with the highest relative blackbody flux show the largest increase in pulse fraction. 


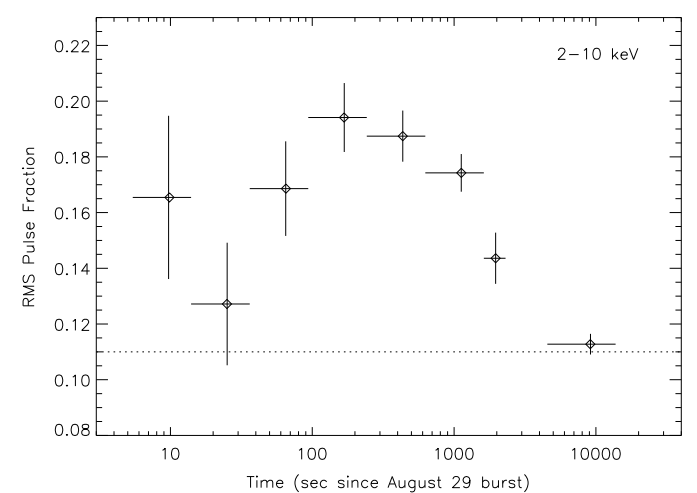

FIGURE 4. The evolution of the $2-10 \mathrm{keV}$ pulse fraction during the X-ray tail following the burst of 1998 August 29. The dotted line denotes the average pulse fraction observed during quiescence.

\section{PULSE PROFILES}

Currently, the pulse profiles of both SGR $1900+14$ and SGR $1806-20$ are very nearly sinusoidal (i.e. they show very little power at the higher harmonics). This has not always been the case, however, as both SGRs have shown significant changes in their pulse profiles during the last several years. The most notable of which was the dramatic change in the pulse profile of SGR $1900+14$ during the tail of the giant flare of August $27[6,7,8,9]$.

Forty seconds after the onset of the August 27 flare, $5.16 \mathrm{~s}$ coherent gamma-ray pulsations at high amplitude emerged. Initially, the pulse profile was complex, having four distinct maxima per rotation cycle. Toward the end of the flare, the pulse profile was significantly more sinusoidal (Figure 5 - middle row). The same qualitative behavior was observed in the persistent X-ray emission from SGR $1900+14$. In all observations prior to 1998 August 27, the pulse profile was complex having significant power at higher harmonics (Figure 5 - top row). For all observations after August 27 through early 2000, the pulse profile remained relatively simple (Figure 5 bottom row). Hence, the pulse profile change observed at gamma-ray energies during the tail of the August 27 flare translated to the persistent emission from this SGR in a sustained manner (i.e. for years after the August 27 X-ray tail had disappeared) [13].

By the middle of the year 2000, the pulse profile began to show slightly more power in the higher harmonics [25]. The next observations of SGR $1900+14$ took place in the hours and days following the April 18 intermediate flare. At some point between the latter half of 2000 and the days directly after the April 18 flare, the pulse profile simplified in shape [24]. As with the August 27 flare, the direction of the pulse profile change here was the same in that power at the higher harmonics lessened following the flare [25].

A systematic study of the temporal and spectral evolution of the pulse profile of SGR $1900+14$ using exclusively RXTE PCA observations has recently been completed [25]. In this study, we show that there is a significant energy dependence of the pulse profile, particularly when the profile was complex in shape (i.e. prior to 1998 August 27). Moreover, the pulsed flux spectrum during the X-ray tail of the August 27 flare becomes harder with time.

The evolution of the pulse profile of SGR 1806-20 is also presented in [25]. We find significant temporal evolution in the pulse profile of this SGR from 1996 November to 1999 January. Due to the sparseness of the observations, however, we cannot determine the exact time of this change, nor the timescale over which it progressed to better than 2.3 years.

\section{PULSE TIMING}

Coherent pulsations from the persistent emission of SGR 1806-20 were discovered within an RXTE PCA observation from 1996 November [15]. From archival observations, it was found that the spin frequency of this SGR was decreasing rapidly, indicative of a strongly magnetized neutron star spinning down via magnetic braking [15]. The spin frequency history of this SGR now extends from 1993 through 2001 (Figure 6 [26]). We have found that at all times, the SGR has been spinning down, but the rate of spindown shows substantial variability. In fact, the measured spin-down torque on this SGR has been found to vary by up to a factor $\sim 4$. Unlike the flux variability of SGR $1900+14$, the torque variations seen in SGR 1806-20 do not correlate with the burst activity [26].

Pulsations from the X-ray counterpart of SGR 1900+14 were discovered during an ASCA observation in 1998 April [27], shortly before the SGR entered an intense, sustained burst active interval [28]. Similar to SGR 1806-20, subsequent observations showed that this SGR was spinning down rapidly and irregularly $[19,29]$. The spin frequency history of this SGR now extends from 1996 through 2001 (Figure 7 [26]). As with SGR 1806-20, the variations in torque do not directly correlate with the burst activity from this SGR with one notable exception, the giant flare of August 27.

From an earlier compilation of pulse frequency measurements between 1996 September and 1999 January, we showed that the spin down of this SGR showed small variations, yet remained constant on average for timescales longer than about a month at nearly all epochs 
Sep 96
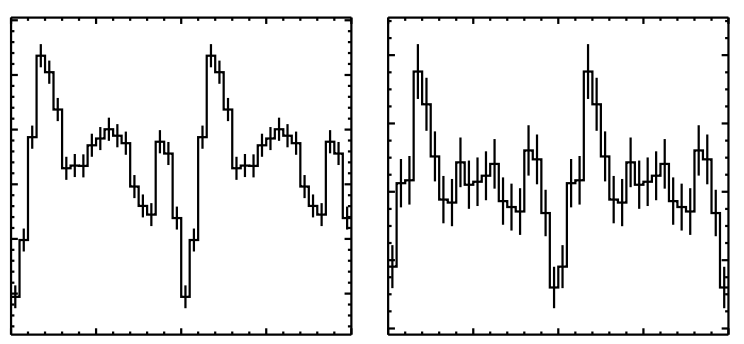

$T_{0}+40 \mathrm{~s}$ to $T_{0}+100 \mathrm{~s}$

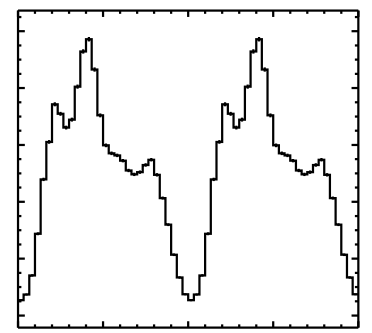

Apr 98

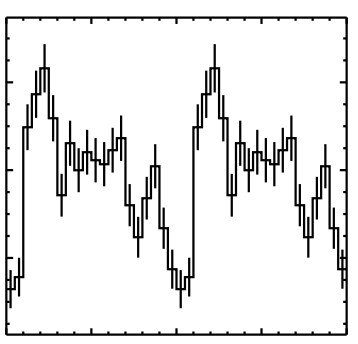

$\mathrm{T}_{\mathrm{o}}+280 \mathrm{~s}$ to $\mathrm{T}_{\mathrm{o}}+330 \mathrm{~s}$
Jun 98

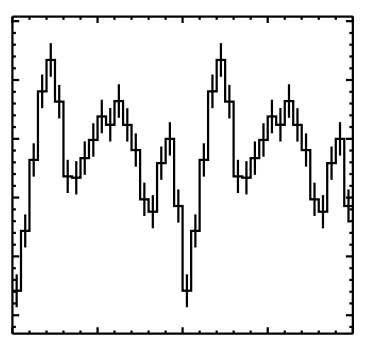

August $27^{\text {th }}$

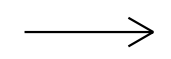

Transition

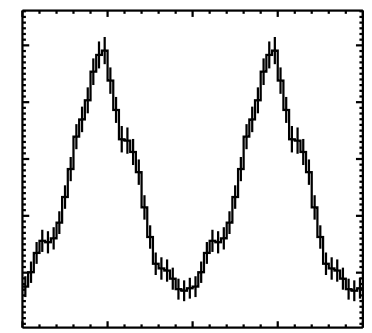

Sep 98

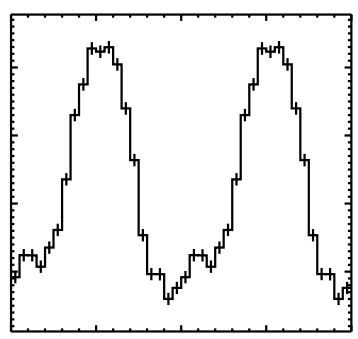

Jan 99

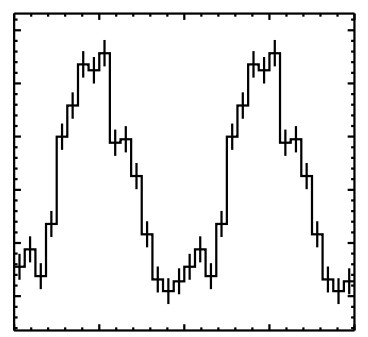

Mar 00

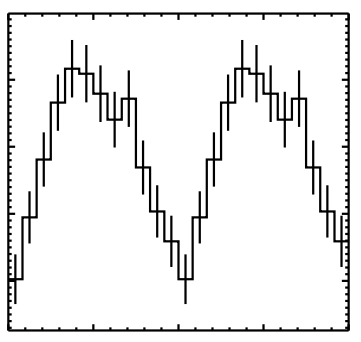

Jun 00

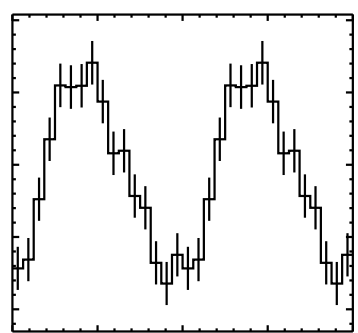

FIGURE 5. Evolution of the pulse profile of SGR 1900+14 covering 3.8 years. All panels display two pulse cycles and the vertical axes are count rates with arbitrary units. The two middle panels were selected from Ulysses data $(25-150 \mathrm{keV})$ of the August $27^{\text {th }}$ flare. Times over which the Ulysses data were folded are given relative to the onset of the flare $\left(T_{0}\right)$. See text for further details. The top and bottom rows are integrated over the energy range $2-10 \mathrm{keV}$. From top-to-bottom, left-to-right, the data were recorded with the RXTE, BeppoSAX, ASCA, RXTE, RXTE, RXTE, BeppoSAX, and RXTE. 

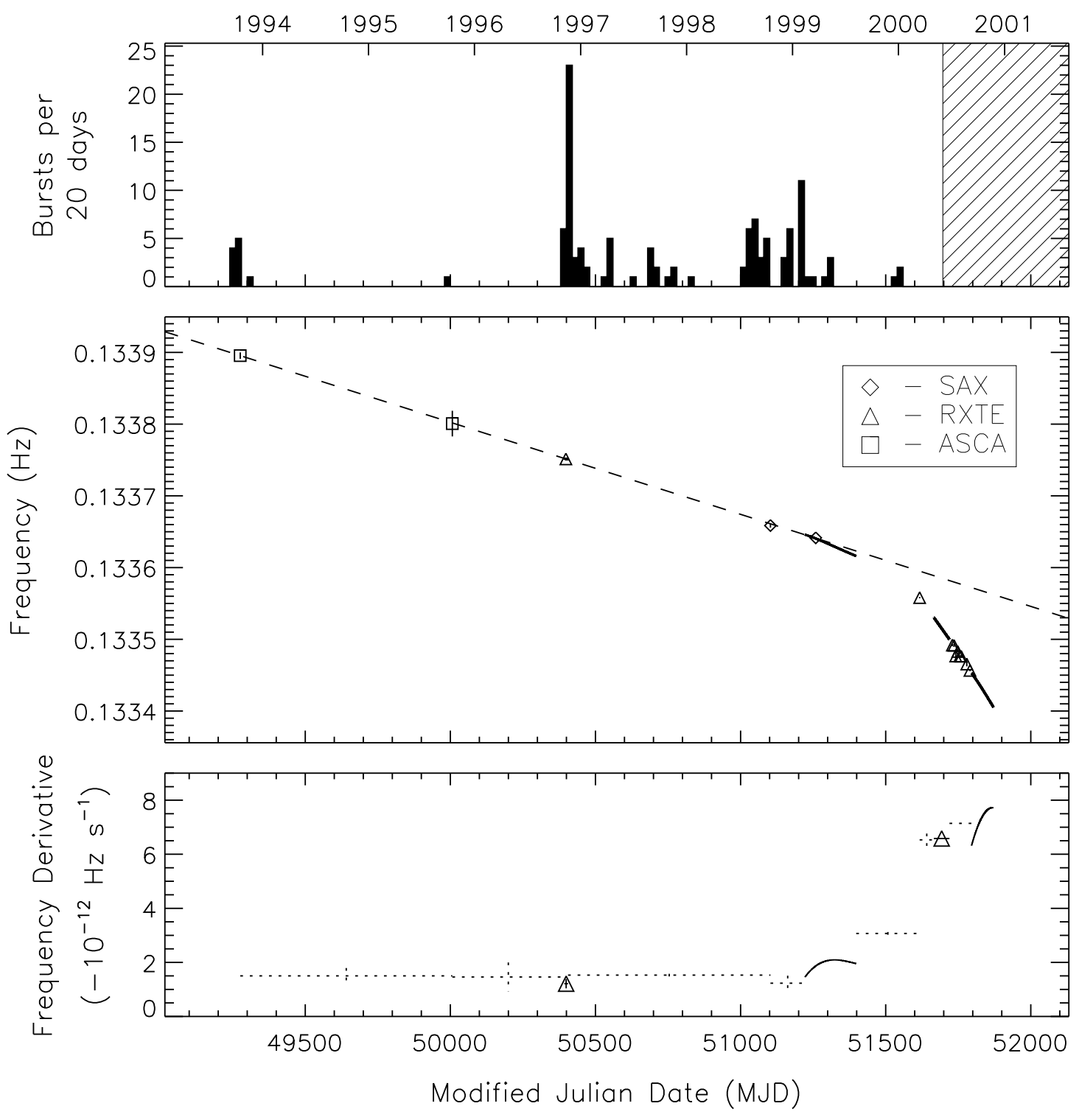

FIGURE 6. Top - Burst rate history of SGR 1806-20 as observed with BATSE. The hashed region starts at the end of the CGRO mission. Middle - The frequency history of SGR 1806-20 covering 7.1 years. Plotting symbols mark individual frequency measurements and solid lines denote phase-connected timing solutions. The dashed line marks the average spin-down rate prior to burst activation in 1998. Bottom - The frequency derivative history over the same timespan. Dotted lines denote average frequency derivative levels between widely spaced frequency measurements. Solid lines mark phase-coherent timing solutions and triangles mark instantaneous torque measurements, both using RXTE PCA data. 

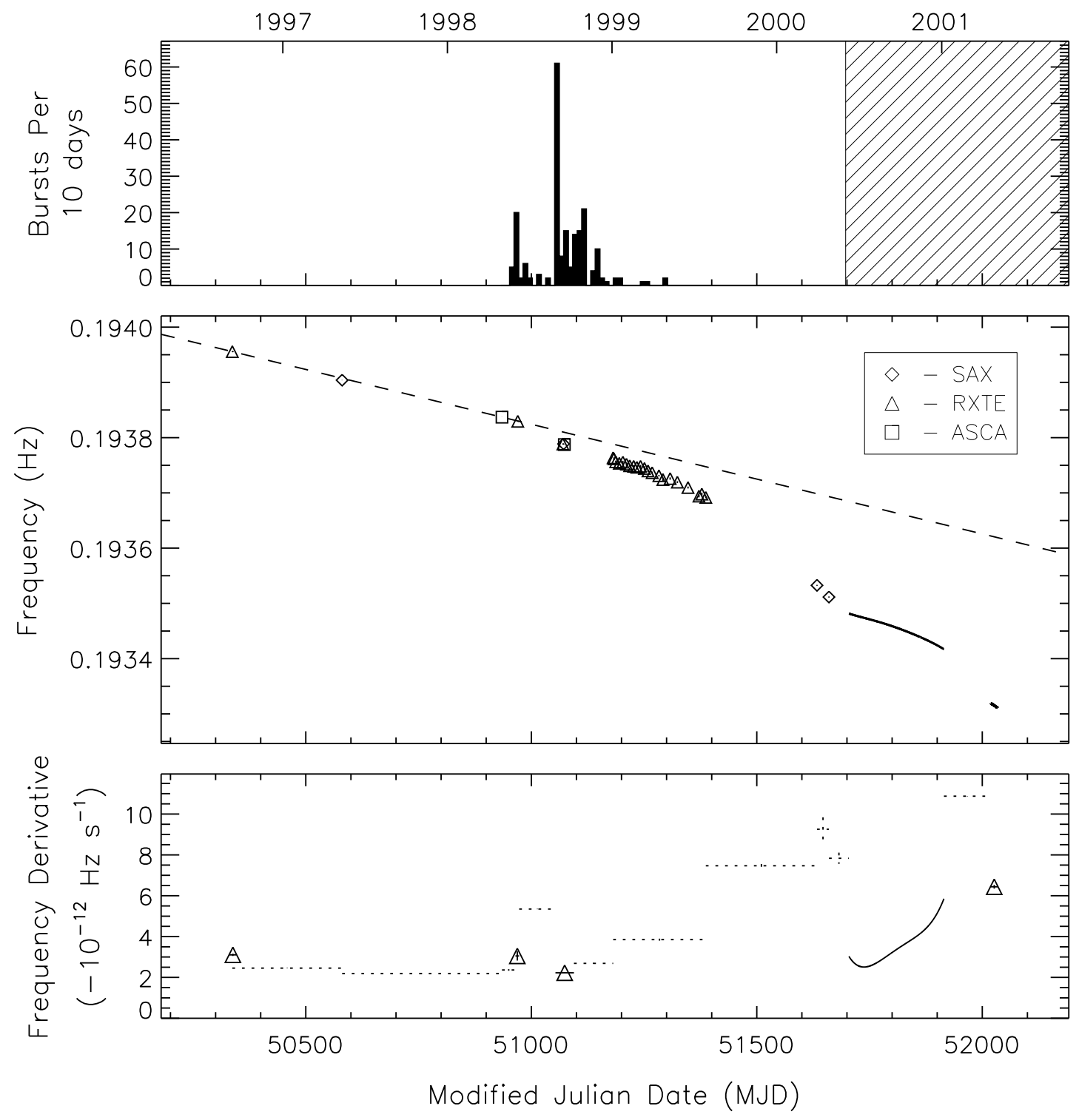

FIGURE 7. Top - Burst rate history of SGR $1900+14$ as observed with BATSE. The hashed region starts at the end of the CGRO mission. Middle - The frequency history of SGR 1900+14 covering 4.7 years. Plotting symbols mark individual frequency measurements and solid lines denote phase-connected timing solutions. The dashed line marks the average spin-down rate prior to burst activation in 1998. Bottom - The frequency derivative history over the same timespan. Dotted lines denote average frequency derivative levels between widely spaced frequency measurements. Solid lines mark phase-coherent timing solutions and triangles mark instantaneous torque measurements, both using RXTE PCA data. 
[29]. The lone exception to this rule was an 80 day interval during the middle of 1998 where the average spindown rate nearly doubled. Contained within this 80 day interval was the August 27 flare and we argued that this spin-down anomaly was likely linked to the flare [29]. Subsequently, Palmer [30] showed that the phase of the pulsations in gamma-rays was offset from a backward extrapolation of the X-ray pulse train recorded during the days following the flare, supporting our earlier inference that the star had spun down rapidly during/after the giant flare. The conclusion of rapid spin down depends critically on the energy dependence of the pulse profile. More recent observations of gamma-ray pulsations during the 2001 April 18 flare have shown that there is very little change in the pulse profile with energy for this source [24], substantiating the claim that the phase offset in the August 27 flare was due to a sudden change in torque, perhaps from a relativistic particle outflow from the stellar surface. Gamma-ray observations during the flare [9] and a transient radio nebula discovered following the flare [31] provide independent evidence for the existence of a particle outflow.

As illuded to in the previous paragraph, we searched for a similar effect in the aftermath of the April 18 intermediate flare. We found that unlike the August 27 flare, the phase of the gamma-ray pulsations matched nicely with the X-ray ephemeris [24]. A fortuitous X-ray monitoring observation 4 days prior to the flare shows that there was a short timescale change in the spin ephemeris somewhere between April 14 and April 18, however, the brevity of the April 14 observation precluded us from constraining the manner in which this change occurred.

Finally, we note that although the August 27 flare likely did alter the spin down of SGR $1900+14$, its impact was very small relative to the much larger variations observed during $\sim 5$ years of monitoring. So, in general, the direct effects of burst activity are insignificant to the overall torque noise in each of these SGRs. For a more complete discussion of the torque variability in these two SGRs, as well as a quantitative analysis of the torque noise, see [26].

\section{DISCUSSION}

We have summarized the recent observations of dynamic behavior in the persistent and pulsed emission from SGR $1900+14$ and SGR 1806-20. Now, we will discuss what constraints these observations place on the models for the SGRs, in particular the magnetar model.

The magnetar model postulates that the SGRs are young neutron stars with super-strong magnetic fields $\left(B \sim 10^{14}-10^{15} \mathrm{G}\right)$. It is the decay of this strong field which powers both the burst and persistent emission
$[32,33]$. The steady X-ray emission is generated by persistent magnetospheric currents and low-level seismic activity within and beneath the stellar surface. The burst emission is due to the build up of stress in the stellar crust from the evolving magnetic field and the eventual release of this stress when the crust fractures. To date, this model provides the most accurate description of the persistent, pulsed, and burst properties of SGRs.

Currently, only four clear X-ray tails have been detected, all from SGR $1900+14$. As mentioned earlier, there is a potential correlation between the relative abundance of thermal emission in the tail and the enhancement of the pulse fraction. This correlation, if proved correct with the detection and analysis of several more SGR tails, would provide a strong argument for heating of a localized region on the neutron star during bursts. Since the pulse fraction increases during some of these tails, the flux enhancement must be anisotropic about the star. In the cases of the August 29 and April 28 burst tails, the location of the heating is also constrained. In each of these events, we have precise pulse phase information prior to, during, and after the tail. For both bursts, the phase of the pulsations during the tail does not shift relative to the pulse phase prior to the burst. This requires that the localized region on the neutron star with the largest relative flux enhancement is the same region giving rise to the persistent X-ray pulse peak (e.g. the polar cap).

With regards to the magnetar model, localized heating of the polar cap requires that the fracture site of the burst be at the same location. The peak of the August 29 flare in gamma-rays lags behind the centroid of the pulse profile peak in X-rays by $\sim 0.1$ cycle, although the burst light curve (duration $\sim 3.5 \mathrm{~s}$ ) covers a large fraction of a pulse cycle spanning the pulse valley. The phase alignment of the April 28 burst is yet to be determined. Measuring the phase alignment of the April 28 burst and detailed modeling of the expected lag between the peak in the X-ray pulse profile and the rise and/or peak of the burst are required before one can determine whether or not these two bursts fit with the picture of a localized fracture region near the polar cap.

The dramatic change in the pulse profile of SGR 1900+14 in conjunction with the giant flare requires a substantial change in the magnetic field of the neutron star [13,34]. In the magnetar model, there are at least two possible ways this can happen. One possibility is that a twist in the magnetosphere is generated following the flare, driving a persistent current which produces an optically thick scattering screen at some substantial distance $\left(\sim 10 R_{*}\right)$ from the stellar surface. In this model, the surface field geometry remains complex at all times. The pulse profile, however, simplifies when the scattering screen is present (i.e. after the flare). The scattering screen must have the properties of redistributing the radiation in phase, but not in energy in order to account 
for the reemergence of the blackbody component after the August 27 tail fades away [35]. The decay of this magnetospheric twist is believed to be several years. An alternative scenario involves restructuring of the surface magnetic field geometry. In this picture, the field geometry is complex prior to the flare and relaxes to a more dipolar structure following the event giving rise to the observed change in pulse profile.

Thompson, Lyutikov \& Kulkarni [35] recently investigated each of these scenarios in detail, noting advantages and disadvantages for each model. In this work, they have identified further observational tests involving the energy spectrum of the emission before and after the flare. Simulations of the expected behavior [36] and an analysis of the spectral evolution of SGR 1900+14 [37] are currently underway to work towards resolving this issue.

Unlike the flux variability, the torque enhancements in these systems do not correlate with the burst activity. In the context of the magnetar model, the abscence of a direct correlation between these two parameters has strong implications for the underlying physics behind each phenomenon. The magnetar model postulates that the bursting activity in SGRs is a result of fracturing of the outer crust of a highly magnetized neutron star. Furthermore, the majority of models proposed to explain the torque variability in magnetars invoke crustal motion and/or low-level seismic activity [38, 39, 34]. Since there is no direct correlation between the burst activity and torque variability, then either $(i)$ the seismic activities leading to each observable are decoupled from one another, or (ii) at least one of these phenomena is not related to seismic activity [26]. Simultaneous spectral information from imaging X-ray telescopes (e.g. BeppoSAX, Chandra, and XMM-Newton) complimentary to the torque measurements obtained with the RXTE PCA would be useful in determining the nature of the torque variabilitity in these SGRs.

\section{ACKNOWLEDGMENTS}

I thank the many collaborators who have contributed to the results discussed here. I would like to thank Chryssa Kouveliotou for many useful discussions and a careful reading of the manuscript. I acknowledge my own support from the Long Term Space Astrophysics program (NAG 5-9350).

\section{REFERENCES}

1. Olive, J.-F., et al., "FREGATE observations of a strong burst from SGR 1900+14", in Woods Hole 2001 GRB Conference, edited by R. Vanderspek, AIP, New York, 2002.

2. $\quad$ Cheng, B., et al., Nature, 382, 518 (1996).

3. Göğüss, E., et al., ApJ, 558, 228 (2001).

4. Bak, P., Tang, C., and Wiesenfeld, K., Phys. Rev. A, 38, 364 (1988).

5. Mazets, E., et al., Nature, 282, 587 (1979).

6. Hurley, K., et al., Nature, 397, 41 (1999).

7. Mazets, E., et al., Astron. Lett., 25, 635 (1999).

8. Feroci, M., et al., ApJ, 515, L9 (1999).

9. Feroci, M., Hurley, K., Duncan, R., and Thompson, C., ApJ, 549, 1021 (2001).

10. Guidorzi, C., et al., GCN Circ. 1041 (2001).

11. Kouveliotou, C., et al., in preparation (2002).

12. Woods, P., et al., ApJ, 518, L103 (1999).

13. Woods, P., et al., ApJ, 552, 748 (2001).

14. Kouveliotou, C., et al., ApJ, 558, L47 (2001).

15. Kouveliotou, C., et al., Nature, 393, 235 (1998).

16. Hurley, K., "The $4.5 \pm 0.5$ Soft Gamma Repeaters in Review", in Gamma-Ray Bursts: $5^{\text {th }}$ Huntsville Symp., edited by R. Kippen, R. Mallozzi, and G. Fishman, AIP 526, New York, 2000, p. 763.

17. Ibrahim, A., et al., ApJ, 558, 237 (2001).

18. Remillard, R., Smith, D., and Levine, A., IAU Circ. 7002 (1998).

19. Kouveliotou, C., et al., ApJ, 510, L115 (1999).

20. Murakami, T., et al., ApJ, 510, L119 (1999).

21. Lenters, G., et al., "An Extended Burst Tail from SGR 1900+14 with a Thermal X-ray Spectrum", in Woods Hole 2001 GRB Conference, edited by R. Vanderspek, AIP, New York, 2002.

22. Feroci, M., et al., in preparation (2002).

23. Fox, D., et al., ApJ submitted (2002).

24. Woods, $\mathrm{P}$., et al., in preparation (2002).

25. Göğüş, E., et al., ApJ, submitted (2002).

26. Woods, P., et al., $A p J$, in press (2002).

27. Hurley, K., et al., ApJ, 510, L111 (1999).

28. Hurley, K., et al., ApJ, 510, L107 (1999).

29. Woods, P., et al., ApJ, 524, L55 (1999).

30. Palmer, D., astro-ph/0103404 (2001).

31. Frail, D., Kulkarni, S., and Bloom, J., Nature, 398, 127 (1999).

32. Thompson, C., and Duncan, R., MNRAS, 275, 255 (1995).

33. Thompson, C., and Duncan, R., ApJ, 473, 322 (1996).

34. Thompson, C., et al., ApJ, 543, 340 (2000).

35. Thompson, C., Lyutikov, M., and Kulkarni, S., ApJ, in press (2002).

36. Thompson, C., et al., in preparation (2002).

37. Woods, P., et al., in preparation (2002).

38. Thompson, C., and Blaes, O., Phys. Rev. D, 57, 3219 (1998).

39. Harding, A., Contopoulos, I., and Kazanas, D., ApJ, 525, L125 (1999). 